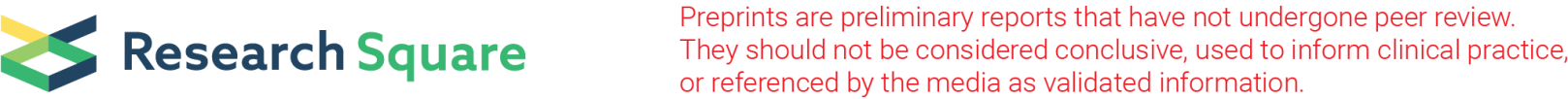

\section{Significance of Plasma Circulating Endothelial Microparticles Combined with Von Willebrand Factor in Coronary Injury of Kawasaki Disease}

\section{Mingye Cheng}

Affiliated Hospital of Nantong University

\section{Tao Chen}

Affiliated Hospital of Nantong University

Jianmei Zhao ( $\nabla$ zjmheart@163.com )

Affiliated Hospital of Nantong University

Zhiyuan Tang

Affiliated Hospital of Nantong University

\section{Research Article}

Keywords: Coronary artery lesions, endothelial microparticles,kawasaki disease, von Willebrand factor.

Posted Date: August 12th, 2021

DOI: https://doi.org/10.21203/rs.3.rs-789668/v1

License: (c) (i) This work is licensed under a Creative Commons Attribution 4.0 International License.

Read Full License 


\section{Abstract}

Background:The greatest complication of Kawasaki disease (KD) is coronary artery injury, and the requirement for early diagnosis and treatment is paramount. Thus, markers of vascular endothelial injury are of important clinical significance.

Methods:According to our diagnostic criteria, blood samples were collected from 43 patients with KD, who were then divided into coronary artery lesions (CALs) and non-CALs (NCALs) groups according to their Z-score. As the control group, an additional 26 blood samples were collected from healthy children. Flow cytometry (FCM) and enzyme-linked immunosorbent assays (ELISA) were used to detect the expression levels of plasma endothelial microparticles (EMPs) and von Willebrand factor (vWF).

Results:The expression levels of plasma CD31+/CD42b-EMPs, CD105+/CD54+EMPs, and vWF were higher in children with KD than those in the control group, and the differences were statistically significant $(P<0.05)$. Also, the expression levels of CD31+/CD42b-EMPs, CD105+/CD54+EMPs and vWF in those in the CALs group at the acute and subacute stages were significantly higher than those in the NCALs group $(P<0.05)$. Furthermore, receiver operating characteristic $(R O C)$ curve analysis revealed that the area under the curve (AUC) of CD31+/CD42b-EMPs combined with vWF was 0.896, which indicates a higher diagnostic value in predicting CALs in children with KD.

Conclusions:In our study, expression levels of EMPs and vWFare expected to used for early diagnosis, and which are associated with coronary artery injury in KD.

\section{Introduction}

Kawasaki disease (KD) is now considered to be one of the most common causes of childhood acquired heart disease in developed countries. Among the cardiovascular complications of KD, coronary artery lesions (CALs) are especially likely to result in long-term morbidity and mortality[1-2]. During KD, the cascade amplification effect of cellular factors causes the infiltration of circulating immune effector cells into blood vessels, leading to vascular endothelial injury [3-4]. The long-term effects of endothelial injury and persistent vascular response damage in children with KD is concerning, irrespective of coronary artery disease status.

The identification of KD patients with CALs is challenging, and the use of predictive biomarkers holds great promise. Circulating endothelial microparticles (EMPs) are small membranous vesicles released from endothelial cells under various stimuli. EMPs can regulate numerous pathophysiological processes, such as thrombosis, angiogenesis, endothelial function and endothelial reactivity [5-6]. During activation and apoptosis, EMPs with different phenotypes are released from endothelial cells in varying quantities. Indeed, with the exception of CD144, most of the remaining surface antigen are not specifically expressed on endothelial cells[7]. In order to improve the detection sensitivity, we ultimately selected two different groups of antigenic markers (CD31+/CD42b- and CD105+/CD54+). CD31 can be expressed on the hils and specific $T$ cell subsets. Under abnormal 
conditions, CD31 can induce angiogenesis and promote cellular migration[8]. CD42b is one of the primary surface glycoproteins of the platelet membrane, though it is not expressed by endothelial cells[9]. CD105 is a transmembrane glycoprotein which is abundantly expressed in the tissue vascular endothelium and is up-regulated in proliferating endothelial cells[10]. CD54 is one of the primary adhesion molecules expressed by vascular endothelial cells. It can result in the activation of several pro-inflammatory signaling cascades and promoting an inflammatory response at the blood vessel wall[11].

von Willebrand factor (VWF) is a glycoprotein polymer present in the plasma and on platelets, and is produced by endothelial cells, megakaryocytes and in the subcutaneous tissue. Under normal circumstances, circulating VWF remains in a resting state. When the body is stimulated, VWF is involved in thrombus formation via multiple different pathways[12-13]. Various studies have suggested that the level of vWF is an indirect measure of the effects of multiple different stimuli on endothelial cells, and that levels can reflect activation or apoptosis of endothelial cells [14].

Studies have also shown that children with KD have obvious endothelial cell dysfunction and a prethrombotic hypercoagulable state, and continuous development of these pathologies can ultimately lead to CALs[15]. EMPs and VWF can activate inflammatory reactions and coagulation mechanisms through different signaling pathways, resulting in endothelial injury. We speculate that EMPs and vWF play an important role in the occurrence of KD, and are involved in the formation of CALs. Therefore in the present study, we evaluated the expression levels of EMPs and VWF in KD patients, and determined their diagnostic value in KD-associated coronary artery injury.

\section{Material And Methods}

\section{Patients and blood samples}

According to the KD diagnostic criteria of American Heart Association (AHA) in 2017[2], we consecutively included 43 children diagnosed and hospitalized in Affiliated Hospital of Nantong University from October 2018 to August 2019. All the children did not treated with intravenous immunoglobulin (IVIG), aspirin or glucocorticoid before admission. Blood samples were obtained from patients at the acute, subacute and convalescent stages (The staging is based on the 8th edition of "Zhufutang Practical Pediatrics"). All the children underwent echocardiography, and the Z-score was calculated according to the examination results. The Z-score calculation method is based on the analysis of the database proposed by Kobayashi et al.[16] (http://raise.umin.jp/zsp/). Patients with the Z-score $\geq 2$ was defined as the CALs group. The clinical information of all KD patients were collected, including gender, age, height, weight, fever duration, time to start IVIG, whether IVIG is unresponsive, whether of using glucocorticoid, white blood counts (WBC), neutrophil ratio (NE\%), platelet (PLT) counts, C-reactive protein (CRP), erythrocyte sedimentation rate (ESR), alanine aminotransferase (ALT). At the same time, we included 26 age-matched ( 6 months $\leq$ age $\leq 5$ years) healthy controls from outpatient department of pediatrics. The study protocol was approved by the Institution Review Board of The Affiliated Hospital of Nantong University. Informed 
Blood samples were drawn into EDTA-K2 anticoagulation vacuum tube $(2 \mathrm{ml})$ and were centrifuged for 10 min at $160 \mathrm{~g}$ to prepare platelet-rich plasma (PRP). The PRP was then centrifuged for 6 min at $1000 \mathrm{~g}$ to prepare platelet-poor plasma (PPP). The $300 \mu \mathrm{L}$ upper layer of PPP and other $300 \mu \mathrm{L}$ PPP were stored in $80^{\circ} \mathrm{C}$ until use.

\section{Flow Cytometry (Fcm)}

The PPP (65uL) in a polypropylene tube was incubated with $20 \mu \mathrm{L}$ of PE anti-CD31 (BD, USA), $20 \mu \mathrm{L}$ of FITC anti-CD42b (BD, USA), 20uL of APC anti-CD54 (BD, USA) and 5 uL PerCP-Cy ${ }^{\text {Tw }} 5.5$ anti-CD105 (BD, USA) for $15 \mathrm{~min}$ in the dark. At the same time, we took another PPP $(65 \mathrm{uL})$ in a polypropylene tube incubated with equal amount of isotype control antibodies for $15 \mathrm{~min}$ in the dark. Then $370 \mu \mathrm{L}$ of PBS was added and the sample was ready for flow cytometry on a FACSCalibur flow cytometer using CellQuest Pro software (BD, USA). EMP levels was defined as the ratio of CD31+/CD42b- EMPs and CD54+/CD105+ EMPs.

\section{Enzyme-linked Immunosorbent Assay (Elisa)}

The plasma vWF levels in children were measured using ELISA kits (Shanghai Enzyme-linked Biotechnology Co., Ltd.) according to the manufacturer's instructions.

\section{Statistical analysis}

Statistical analyses were conducted with SPSS software. The measurement data are expressed as mean \pm standard deviation ( $\nabla x \pm s$ ). Comparisons between 2 groups were carried out using $t$ test, and those among multiple groups were carried out using one-way ANOVA. Chi-square test was used for counting data, and Spearman's rank correlation analysis was used for the correlation of each index. Independent risk factors of CALs in children with KD were predicted by binomial multivariate Logistic regression analysis. Receiver operating characteristic curve (ROC) was drawn and area under curve (AUC) was calculated to evaluate the efficacy of association index in predicting CALs. The significance level was two-sided and set at $\mathrm{P}<0.05$.

\section{Results}

\section{Plasma EMP levels}

The expression levels of plasma CD31+/CD42b-EMPs and CD105+/CD54 + EMPs in KD children at the acute, subacute and convalescent stages were significantly higher than those in the control group $(\mathrm{P}<$ 0.05) (Fig. 2a-b). The levels of CD31+/CD42b- and CD105+/CD54 + EMPs in CALs group patients at the acute and subacute stages were higher than those in the NCALs group, and the differences between the 
two groups were statistically significant $(P<0.05)$. There were no significant differences in the levels of these indicators between the two groups in patients at the convalescent stage $(P>0.05)$ (Fig. 2c-d).

\section{Plasma Vwf Levels}

The expression levels of plasma VWF in KD children at the acute, subacute and convalescent stages were higher than those in the control group, and these results were statistically significant $(P<0.05)$ (Fig. 3a). Furthermore, plasma vWF levels of those in the CALs group at the acute and subacute stages were significantly higher than those in the NCALs group $(P<0.05)$. However, there were no significant differences at the convalescent stage $(P>0.05)$ (Fig. 3b).

\section{Correlation analysis of plasma EMPs, vWF and laboratory indicators (WBC, NE\%, PLT, ALT, CRP, ESR)}

The results of Spearman's rank correlation analysis(Table 1) showed that the levels of CD31+/CD 42bEMPs in KD patients at acute stage was positively correlated with the NE\% $(r=0.459, P=0.024)$, and the levels of CRP $(r=0.459, P=0.024)$. The levels of CD105+/CD54 + EMPs were also positively correlated with the NE\% $(r=0.438, P=0.032)$ and the CRP levels $(r=0.437, P=0.033)$, and the levels of vW were positively correlated with those of CRP $(r=0.457, P=0.025)$.

Table 1

Correlation analysis of plasma EMPs, vWF and laboratory test indexes of KD patients at the acute stage

\begin{tabular}{|c|c|c|c|c|c|c|}
\hline & \multicolumn{2}{|c|}{ CD31+/CD42b-EMPs(\%) } & \multicolumn{2}{|c|}{ CD105+/CD54 + EMPs(\%) } & \multicolumn{2}{|c|}{ vWF(ng/ml) } \\
\hline \multicolumn{7}{|c|}{ Laboratory indexes } \\
\hline & $r$ & P-value & r & P-value & $r$ & P-value \\
\hline $\operatorname{WBC}\left(10^{9} / \mathrm{L}\right)$ & 0.360 & 0.084 & 0.391 & 0.059 & 0.383 & 0.065 \\
\hline NE (\%) & 0.459 & 0.024 & 0.438 & 0.032 & 0.350 & 0.093 \\
\hline $\operatorname{PLT}\left(10^{9} / \mathrm{L}\right)$ & -0.038 & 0.861 & -0.048 & 0.823 & -0.332 & 0.113 \\
\hline $\operatorname{ALT}(\mathrm{U} / \mathrm{L})$ & 0.253 & 0.233 & 0.049 & 0.821 & 0.224 & 0.294 \\
\hline CRP (mg/L) & 0.506 & 0.012 & 0.437 & 0.033 & 0.457 & 0.025 \\
\hline $\operatorname{ESR}(\mathrm{mm} / \mathrm{h})$ & 0.155 & 0.469 & 0.129 & 0.549 & 0.140 & 0.513 \\
\hline
\end{tabular}

\section{Risk Factors For Cals Were Analyzed Based On Z-value}

KD patients were assigned to the CALs or NCALs group according to their Z-score, and the risk factors for CALs were a fever duration $>10$ days, no response to IVIG and CRP $\geq 100 \mathrm{mg} / \mathrm{I}$ (Table 2). 
Table 2

Risk factors for KD complicated with CALs at the acute stage

\begin{tabular}{|c|c|c|c|c|c|}
\hline Parameter & $\mathbf{n}$ & CAL & NCAL & $x^{2}$ & $\mathbf{P}$ \\
\hline & & $(n=11)$ & $(\mathrm{n}=32)$ & & \\
\hline \multicolumn{6}{|l|}{ Sex } \\
\hline male & 22 & $7(63.6)$ & $15(46.9)$ & 0.92 & 0.337 \\
\hline female & 21 & $4(36.4)$ & $17(53.1)$ & & \\
\hline \multicolumn{6}{|l|}{ Age(years) } \\
\hline$<1$ & 9 & $4(36.4)$ & $5(15.6)$ & 2.311 & 0.315 \\
\hline $1 \otimes 5$ & 27 & $6(54.5)$ & $21(65.6)$ & & \\
\hline$>5$ & 7 & $1(9.1)$ & $6(18.8)$ & & \\
\hline \multicolumn{6}{|c|}{ Fever duratio(days) } \\
\hline$\leq 10$ & 39 & $8(72.7)$ & $31(96.9)$ & 5.65 & 0.017 \\
\hline$>10$ & 4 & $3(27.3)$ & $1(3.1)$ & & \\
\hline \multicolumn{6}{|c|}{ Course of disease when IVIG is enabled(days) } \\
\hline$\leq 10$ & 41 & 10(90.9) & $31(96.9)$ & 0.657 & 0.418 \\
\hline$>10$ & 2 & $1(9.1)$ & $1(3.1)$ & & \\
\hline \multicolumn{6}{|c|}{ Non-respond to IVIG } \\
\hline Yes & 6 & $4(36.4)$ & $2(6.2)$ & 6.183 & 0.013 \\
\hline No & 37 & $7(63.6)$ & $30(93.8)$ & & \\
\hline \multicolumn{6}{|c|}{ Glucocorticoid using } \\
\hline Yes & 1 & $0(0.0)$ & $1(3.1)$ & 0.352 & 0.553 \\
\hline No & 42 & $11(100.0)$ & $31(96.9)$ & & \\
\hline \multicolumn{6}{|c|}{ WBC( $\left.10^{9} / L\right)$} \\
\hline$<10$ & 11 & $4(36.4)$ & $7(21.9)$ & 0.914 & 0.633 \\
\hline $10 \rrbracket 20$ & 27 & $6(54.5)$ & $21(65.6)$ & & \\
\hline$>20$ & 5 & $1(9.1)$ & $4(12.5)$ & & \\
\hline \multicolumn{6}{|l|}{$\mathrm{NE}(\%)$} \\
\hline$<80$ & 30 & $6(54.5)$ & $24(75.0)$ & 1.624 & 0.203 \\
\hline
\end{tabular}




\begin{tabular}{|llllll|}
\hline Parameter & $\mathbf{n}$ & CAL & NCAL & $\chi^{2}$ & P \\
\hline$\geq 80$ & 13 & $5(45.5)$ & $8(25.0)$ & & \\
PLT(109/L) & & & & & \\
$<300$ & 20 & $4(36.4)$ & $16(50.0)$ & 0.612 & 0.434 \\
$\geq 300$ & 23 & $7(63.6)$ & $16(50.0)$ & & \\
CRP(mg/L) & & & & & \\
\hline$<100$ & 33 & $5(45.5)$ & $28(87.5)$ & 8.108 & 0.004 \\
$\geq 100$ & 10 & $6(54.5)$ & $4(12.5)$ & & \\
\hline ESR(mm/h) & & & & & \\
\hline$\leq 100$ & 41 & $10(90.9)$ & $31(96.9)$ & 0.657 & 0.418 \\
\hline$>100$ & 2 & $1(9.1)$ & $1(3.1)$ & & \\
\hline ALT(U/L) & & & & & \\
\hline$\leq 45$ & 32 & $7(63.6)$ & $25(78.1)$ & 0.903 & 0.342 \\
\hline$>45$ & 11 & $4(36.4)$ & $7(21.9)$ & & \\
\hline
\end{tabular}

\section{Logistic Regression Analysis}

According to the above results, the plasma CD31+/CD42b- EMPs, CD105+/CD54 + EMPs and vWF values in KD patients at the acute stage were significantly different between the CALs and the NCALs groups ( $P$ $<0.05)$. The model was assessed using the Hosmer Lemeshow test $(P>0.10)$ and the model fitting effect was good. The data (Table 3 ) showed that the plasma CD31+/CD42b- EMPs ratio and the vWF levels were independent risk factors for CALs.

Table 3

Binomial multivariate logistic regression analysis.

\begin{tabular}{|lllllll|}
\hline Parameter & $\mathbf{b}$ & $\boldsymbol{\beta}$ & $\boldsymbol{\chi}^{2}$ & P-value & $\mathbf{9 5 \%} \mathrm{Cl}$ & \\
\hline CD31+/CD42b-EMPs & 0.301 & 0.14 & 4.594 & 0.032 & 1.026 & 1.779 \\
\hline CD105+/CD54 + EMPs & 0.07 & 0.115 & 0.373 & 0.541 & 0.857 & 1.343 \\
vWF & 0.034 & 0.015 & 5.375 & 0.020 & 1.005 & 1.066 \\
constant & -23.236 & 8.470 & 7.526 & 0.006 & & \\
\hline
\end{tabular}

\section{ROC curve analysis}


Subsequently, the CD31+/CD42b-EMPs and vWF values were further analyzed, and ROC curves were constructed for CALs diagnosis. The results (Table 4 and Fig. 4) revealed that the AUC for CD31+/CD42bEMPs was 0.814 [95\% confidence interval $(\mathrm{Cl})=0.674 \sim 0.954$; cutoff value $=27.20 \%$ ], and 0.830 for vWF $(95 \% \mathrm{Cl}=0.685 \sim 0.975$; cutoff value $=283.49 \mathrm{ng} / \mathrm{ml})$; the sensitivity and specificity of predicting CALs were 81.8 and $75.0 \%$ respectively. The AUC for CD31+/CD42b- EMPs combined with vWF was 0.896 (95\% $\mathrm{Cl}=0.819 \sim 0.994)$, and the sensitivity and specificity were 90.9 and $78.1 \%$ respectively. These results indicate that CD31+/CD42b- EMPs combined with vWF are of higher diagnostic value for the prediction of CALs $(P<0.001)$ than either of the two factors alone.

Table 4

ROC curve analysis

\begin{tabular}{|llllll|}
\hline Parameter & AUC & $\boldsymbol{\beta}$ & P-value & $95 \% \mathrm{Cl}$ & \\
\hline CD31+/CD42b-EMPs & 0.814 & 0.071 & 0.002 & 0.674 & 0.954 \\
\hline vWF & 0.830 & 0.074 & 0.001 & 0.685 & 0.975 \\
\hline CD31+/CD42b-EMPs + vWF & 0.906 & 0.045 & $<0.001$ & 0.819 & 0.994 \\
\hline
\end{tabular}

\section{Discussion}

Here, we show relatively high expression levels of EMPs and vWF in the plasma of children at different stages of $\mathrm{KD}$, as well as their roles in CALs formation .

Numerous studies have linked EMPs to a variety of different vascular diseases, such as severe hypertension, acute lung injury, and acute coronary syndrome[17-19]. CD31+/CD42b-is a group of classic marker combinations used to identify EMPs. The current studies found that the levels of CD31+/CD42b-EMPs were elevated in various immune inflammatory pathologies and cardiocerebrovascular diseases which lead to the apoptosis or endothelial cell activation[20-21]. In addition, Yu et al.[22] revealed that the expression levles of CD31 and CD105 by EMPs were significantly increased following apoptotic stimulation, while the levels of CD54, CD62E and CD106 were significantly increased during the activation. The similar results of this study showed that the expression levels of CD31+/CD42b- and CD105+/CD54 + EMPs in patients with acute, subacute and convalescent stage KD were found to be significantly higher than those in the control group $(P<0.05)$. The levels of EMPs were also decreased after treatment, compared with the pre-treatment values, which indicated that the activation and apoptosis of endothelial cells occurred during the development of KD.In the absence of vascular injury, vWF does not interact with circulating platelets[23]. However, during injury, vWF is able to bind to components of the subendothelial connective tissue, promoting the adhesion of platelets to collagen, and thus inducing platelet aggregation[24]. Therefore, theincreased level of vWF in the blood is considered to be an indicator of endothelial injury. Some scholars believe that the high level of vWF in patients with KD reflects a significant acute phase response[25]. In this study, the results illustrated that the levels of VWF in the acute, subacute and convalescent stages of KD was significantly higher than that 
observed in the acute stage, with a downward expression trend following treatment. vWF may serve as a marker of KD and reflect the level of inflammation in the acute stage .

At the same time, this study also defined CALs according to the Z-score. The Z-scores of the coronary artery which adjusted by body surface area were more suitable as a reference standard to objectively describe coronary arteries in children with KD, which may improve the early recognition rate of CALs. The comparison results of expression levels of EMPs and VWF in the CALs and NCALs groups suggested that EMPs and vWF may be involved in the formation of CALs. Spearman's rank correlation analysis were used to assess the association between plasma EMPs, vWF and several clinical laboratory indexes in the acute stage of KD. The EMPs levels were positively correlated with the NE\% and the CRP value. The vWF level was also positively correlated with CRP. CRP is able to stimulate the production of EMPs by human umbilical vein endothelial cells in vitro[26], and other studies have found that an increase in vWF is positively correlated with an increase in inflammatory markers such as CRP and interleukin-6[27]. Similar results were obtained in the this study. Thus we speculated that the aforementioned factors may be involved in KD-associated inflammation in the acute phase. By means of combining the results we concluded that EMPs and VWF were associated with the development and progression of coronary artery injury in KD.

In this study, we collected the clinical data of these $43 \mathrm{KD}$ patients, and analyzed the clinical risk factors for developing CALs. Yamashita et al.[28] retrospectively analyzed the data of 31380 children with KD, and concluded that being male and $<1$ year old, late hospital admission, atypical cases and an IVIG nonresponse were significant risk factors for CALs formations. Furthermore, Flores-Montes et al.[29] suggested that anemia, and being male and $<2$ years old were risk factors for CAL. Other previous studies have concluded that an increase in CRP and ESR, and a decrease in albumin (ALB) increase the risk of developing CALs[30-31]. In future studies, additional clinical data needs to be collected and evaluated to support our conclusions. It is interesting to note that auxiliary examinations should be conducted at the earliest possible opportunity. For children who show indicator risk factors, we should make the additional monitoring of meaningful laboratory indicators for effective evaluation which may reduce the number of missed diagnosis. At the same time, more frequent echocardiography should be performed to evaluate the coronary arteries in a timely manner.

At present, there is no specific global laboratory index for the diagnosis of KD. In the clinic, atypical manifestations frequently result in missed diagnosis and misdiagnosis, and treatment untimely results in cardiovascular complications in the later stage; therefore, early diagnosis is very important for children with KD. Binomial multivariate logistic regression analysis was used to analyze CD31+/CD42b-EMPs, CD105+/CD54 + EMPs and vWF levels, which revealed that the levels of CD31+/CD42b-EMPs and vWF were independent factors for predicting coronary artery injury in patients with KD. A ROC curve was generated to evaluate the diagnostic efficiency of combined and single indexes to predict the efficiency of CALs diagnosis. The results showed that the AUC value of the combined indexes was higher than that of the two individual indexes, and that the sensitivity and specificity were also higher. It can therefore be 
concluded that the combined detection of CD31+/CD42b-EMPs and VWF can improve the sensitivity and specificity of KD-associated CALs diagnosis.

In summary, the results of the present study indicate that EMPs and vWF are significantly increased in the acute stage of KD, particularly in patients with CALs. The increase in EMPs and VWF may be associated with the development and progression of coronary artery injury in KD. The combined evaluation of the above two indexes has high diagnostic value, and can be used as an effective predictor of CALs formation in children with KD.

\section{Conclusion}

The present paper aims to find out the potential early diagnosis indicators in coronary artery injury process for KD. The results of the present study indicated that EMPs and vWF were significantly increased in the acute stage of KD, particularly in patients with CALs. The increasing in EMPs and vWF may be associated with the development and progression of coronary artery injury in KD. The combined evaluation of the above two indexes has high diagnostic value, and can be used as an effective predictor of CALs formation in children with KD.

\section{Declarations}

\section{Funding}

Nantong City Social and People's Livelihood Science and Technology Key Projects (MS22019001); The Scientific Research Program of Jiangsu Province Health Department (H201423); Jiangsu Province Maternal and Child Health Research Projects in key disciplines (F2017510FXK2017041).

\section{Conflicts of interest}

The authors declare that they have no potential conflict of interest.

\section{Availability of data and material}

The datasets used or analysed during the current study are available from the corresponding author on reasonable request.

\section{Code availability}

Not applicable

\section{Authors' contributions}

Conception and design: Jianmei Zhao, Zhiyuan Tang. Experiment performing, data collection, data analyze and manuscript:Mingye Cheng, Tao Chen. All authors have read andapproved the final version of 


\section{Ethics approval}

This study has been approved by the Ethics Committee of the Affiliated Hospital of Nantong University (review number: 2018-K021).

\section{Consent for publication}

Written informed consent for publication was obtained from all participants.

\section{References}

1. Dionne A, Burns JC, Dahdah N, et al. Treatment Intensification in Patients With Kawasaki Disease and Coronary Aneurysm at Diagnosis. Pediatrics. 2019;143(6):e20183341.

2. McCrindle BW, Rowley AH, Newburger JW, et al. Diagnosis, Treatment, and Long-Term Management of Kawasaki Disease: A Scientific Statement for Health Professionals From the American Heart Association. Circulation. 2017;135(17):e927-e999.

3. Sundel RP. Kawasaki disease. Rheum Dis Clin North Am. 2015;41(1):63-viii.

4. Kim KY, Kim DS. Recent Advances in Kawasaki Disease. Yonsei Med J. 2016;57(1):15-21.

5. Rana D, Kumar A, Sharma S. Endothelial Progenitor Cells as Molecular Targets in Vascular Senescence and Repair. Curr Stem Cell Res Ther. 2018;13(6):438-446.

6. McCarthy EM, Wilkinson FL, Parker B, Alexander MY. Endothelial microparticles: Pathogenic or passive players in endothelial dysfunction in autoimmune rheumatic diseases?VasculPharmacol. 2016;86:71-76.

7. Deng F, Wang S, Zhang L. Endothelial microparticles act as novel diagnostic and therapeutic biomarkers of circulatory hypoxia-related diseases: a literature review. J Cell Mol Med. 2017;21(9):1698-

8. Lertkiatmongkol P, Liao D, Mei H, Hu Y, Newman PJ. Endothelial functions of platelet/endothelial cell adhesion molecule-1 (CD31). CurrOpinHematol. 2016;23(3):253-259.

9. Bruyndonckx L,Hoymans VY,Frederix G,et al.Endothelial progenitor cells and endothelial microparticles are independent predictors of endothelial function. The Journal of pediatrics.2014; 165(2):300-305.

10. Kasprzak A, Adamek A. Role of Endoglin (CD105) in the Progression of Hepatocellular Carcinoma and Anti-Angiogenic Therapy. Int J Mol Sci. 2018;19(12):3887.

11. Espagnolle N, Balguerie A, Arnaud E, et al. CD54-Mediated Interaction with Pro-inflammatory Macrophages Increases the Immunosuppressive Function of Human Mesenchymal Stromal Cells. Stem cell reports. 2017; 8(4): 961-976.

12. Shahidi M. Thrombosis and von Willebrand Factor. AdvExp Med Biol. 2017;906:285-306.

13. Yee A, Kretz CA. Von Willebrand factor: form for function. SeminThrombHemost. 2014;40(1):17-27. 
14. Ruggeri ZM, Mendolicchio GL. Interaction of von Willebrand factor with platelets and the vessel wall. Hamostaseologie. 2015;35(3):211-224.

15. Cameron SA, Carr M, Pahl E, DeMarais N, Shulman ST, Rowley AH. Coronary artery aneurysms are more severe in infants than in older children with Kawasaki disease. Arch Dis Child. 2019;104(5):451455.

16. Kobayashi T, Fuse S, Sakamoto N, et al. A New Z Score Curve of the Coronary Arterial Internal Diameter Using the Lambda-Mu-Sigma Method in a Pediatric Population. J Am SocEchocardiogr. 2016;29(8):794-801.

17. Cabrera-Benítez NE, Valladares F, García-Hernández S, et al. Altered Profile of Circulating EndothelialDerived Microparticles in Ventilator-Induced Lung Injury. Crit Care Med. 2015;43(12):e551-e559.

18. Mavroudis CA, Eleftheriou D, Hong Y, et al. Microparticles in acute coronary syndrome. Thromb Res. 2017;156:109-116.

19. Nakaoka H,Hirono K,Yamamoto S,et al.MicroRNA-145-5p and microRNA-320a encapsulated in endothelial microparticles contribute to the progression of vasculitis in acute Kawasaki Disease.Scientific reports,2018; 8(1):1016.

20. Liu Y, Zhang R, Qu H, et al. Endothelial microparticles activate endothelial cells to facilitate the inflammatory response. Molecular medicine reports. 2017; 15(3): 1291-1296.

21. Atehortúa L,Rojas $M$,Vásquez G,et al.Endothelial activation and injury by microparticles in patients with systemic lupus erythematosus and rheumatoid arthritis.Arthritis research \& therapy.2019;21(1):34.

22. Yu X,Xu J,Huang G,et al.Bubble-Induced Endothelial Microparticles Promote Endothelial Dysfunction.PloS one.2017;12(1):e0168881.

23. Jalaer I, Tsakiris DA, Solecka-Witulska BA. The role of von Willebrand factor in primary haemostasis under conditions of haemodilution. Thrombosis research. 2017; 157: 142-146.

24. Wu Q, Hu Y, Jiang M, Wang F, Gong G. Effect of Autophagy Regulated by Sirt1/FoxO1 Pathway on the Release of Factors Promoting Thrombosis from Vascular Endothelial Cells. Int J Mol Sci. 2019;20(17):4132.

25. Jakob A,Schachinger E,Klau S,et al.Von Willebrand factor parameters as potential biomarkers for disease activity and coronary artery lesion in patients with Kawasaki disease.European journal of pediatrics.2020;179(3):377-384.

26. Wang JM,Wang Y,Huang JY,et al.C-Reactive protein-induced endothelial microparticle generation in HUVECs is related to BH4-dependent NO formation.Journal of vascular research.2007;44(3):241-248.

27. Bugała K,Mazurek A,Gryga K,et al.Influence of autoimmunity and inflammation on endothelial function and thrombosis in systemic lupus erythematosus patients.Clinical rheumatology.2018;37(8):2087-2093.

28. Yamashita M,Ae R,Yashiro M,et al.Difference in Risk Factors for Subtypes of Acute Cardiac Lesions Resulting from Kawasaki Disease.Pediatric cardiology.2017;38(2):375-380. 
29. Flores-Montes OA,Valle-Leal J,Arreguin-Reyes R.Risk factors related to cardiovascular complications in patients diagnosed with Kawasaki disease in northwestern Mexico.Boletínmédicodel Hospital Infantil de México.2018;75(3):145-152.

30. Liu MY, Liu HM, Wu CH, et al. Risk factors and implications of progressive coronary dilatation in children with Kawasaki disease. BMC Pediatr. 2017;17(1):139.

31. Yan F,Pan B,Sun H,et al.Risk Factors of Coronary Artery Abnormality in Children With Kawasaki Disease: A Systematic Review and Meta-Analysis.Frontiers in pediatrics.2019;7:374.

\section{Figures}



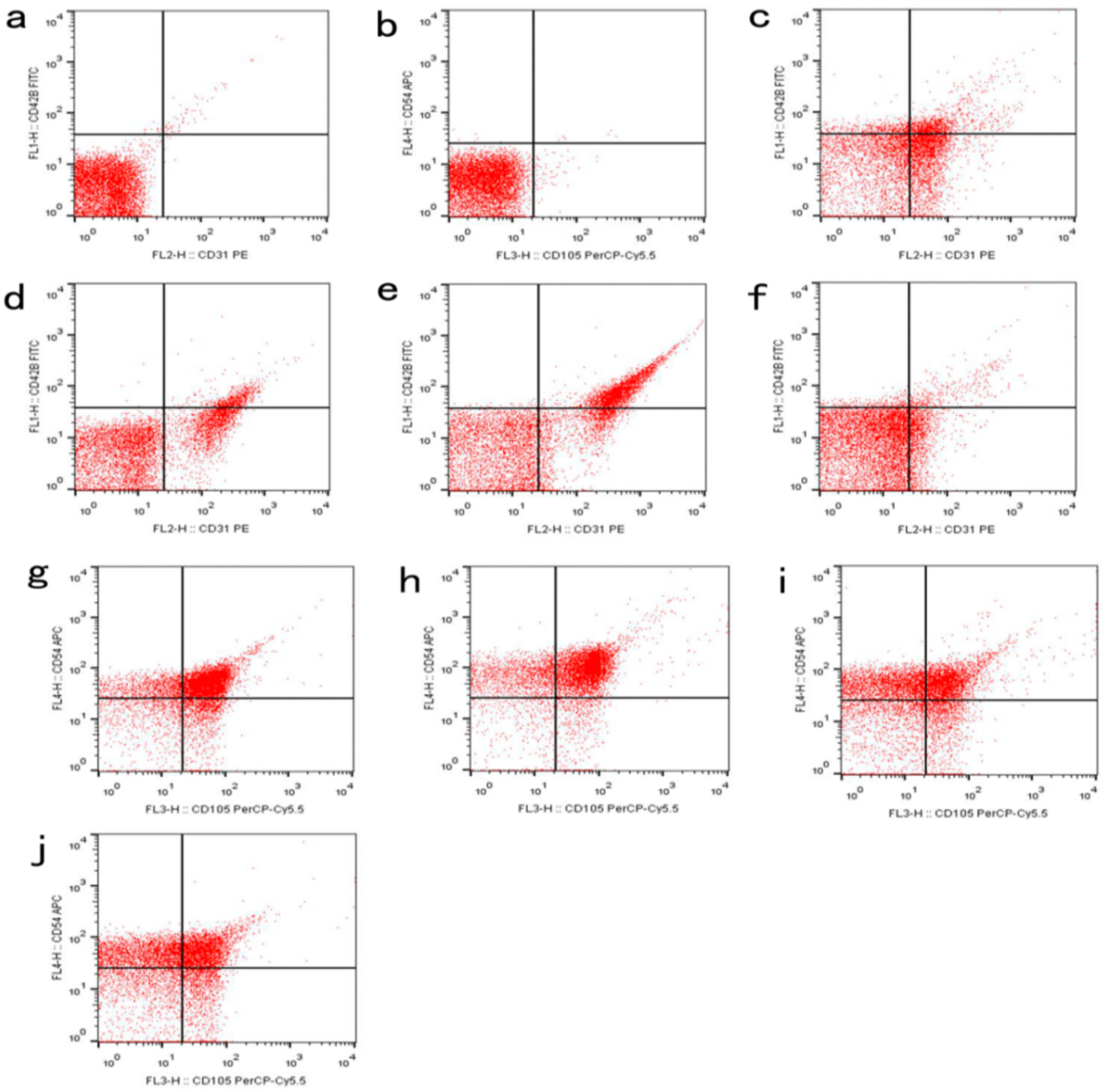

\section{Figure 1}

(a-b) isotype control of CD31/CD42b and CD105/CD54. (c-f) The lower right area shows the CD31+/CD42b-EMPs in KD children at the acute, subacute and convalescent stages and control group. (g-j) The upper right area shows CD105+/CD54+EMPs in KD children at the acute, subacute and convalescent stages and control group. 

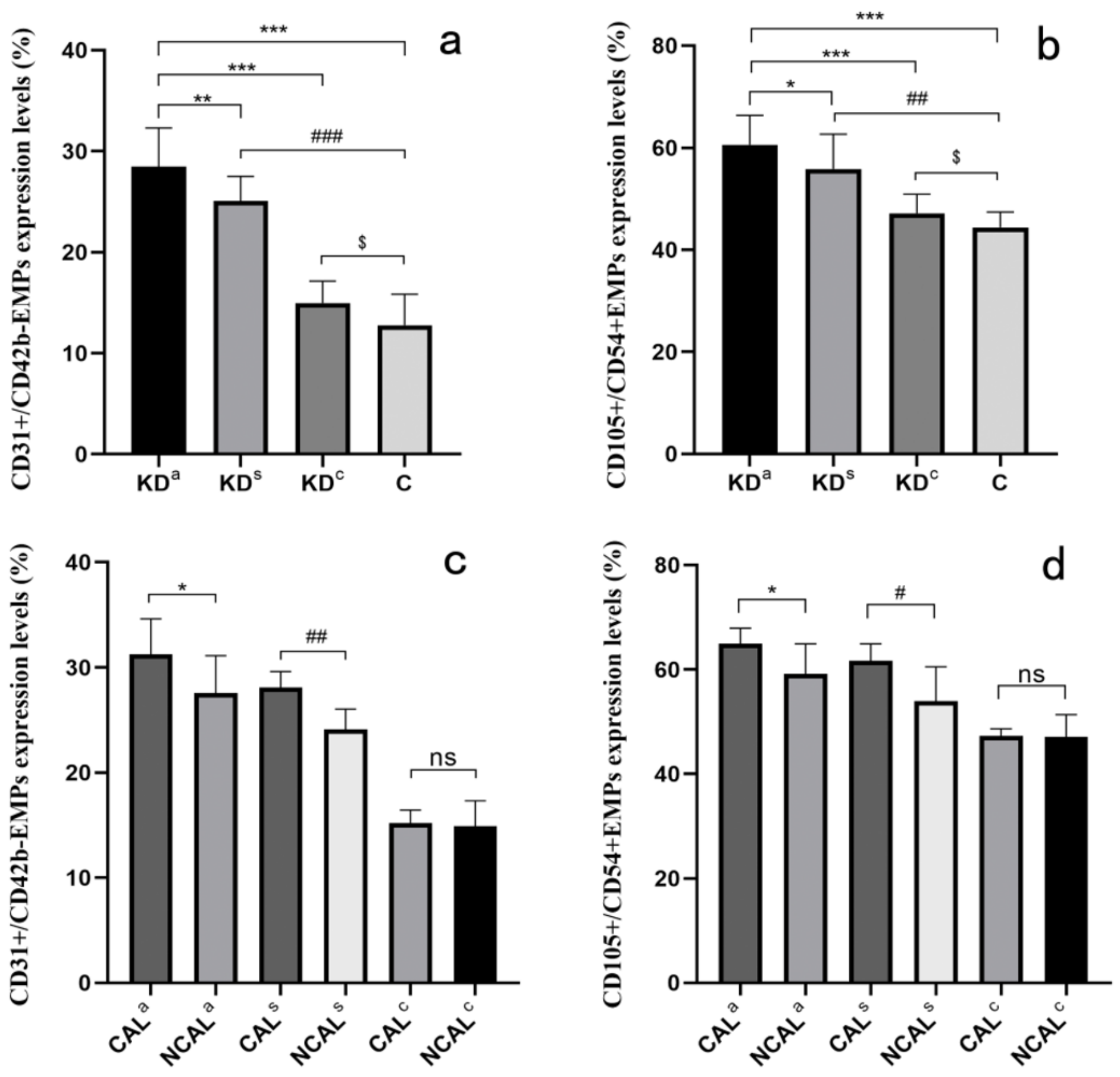

Figure 2

(a-b) Comparison of plasma CD31+/CD42b- and CD105+/CD54+EMPs expression levels (\%) between different groups. (c-d) Comparison of plasma CD31+/CD42b- and CD105+/CD54+EMPs expression levels (\%) between KD groups. */\#/\$P『0.05, **/\#\#/

$$
\mathrm{P} \square 0.01, * * * / \# \# \# /
$$



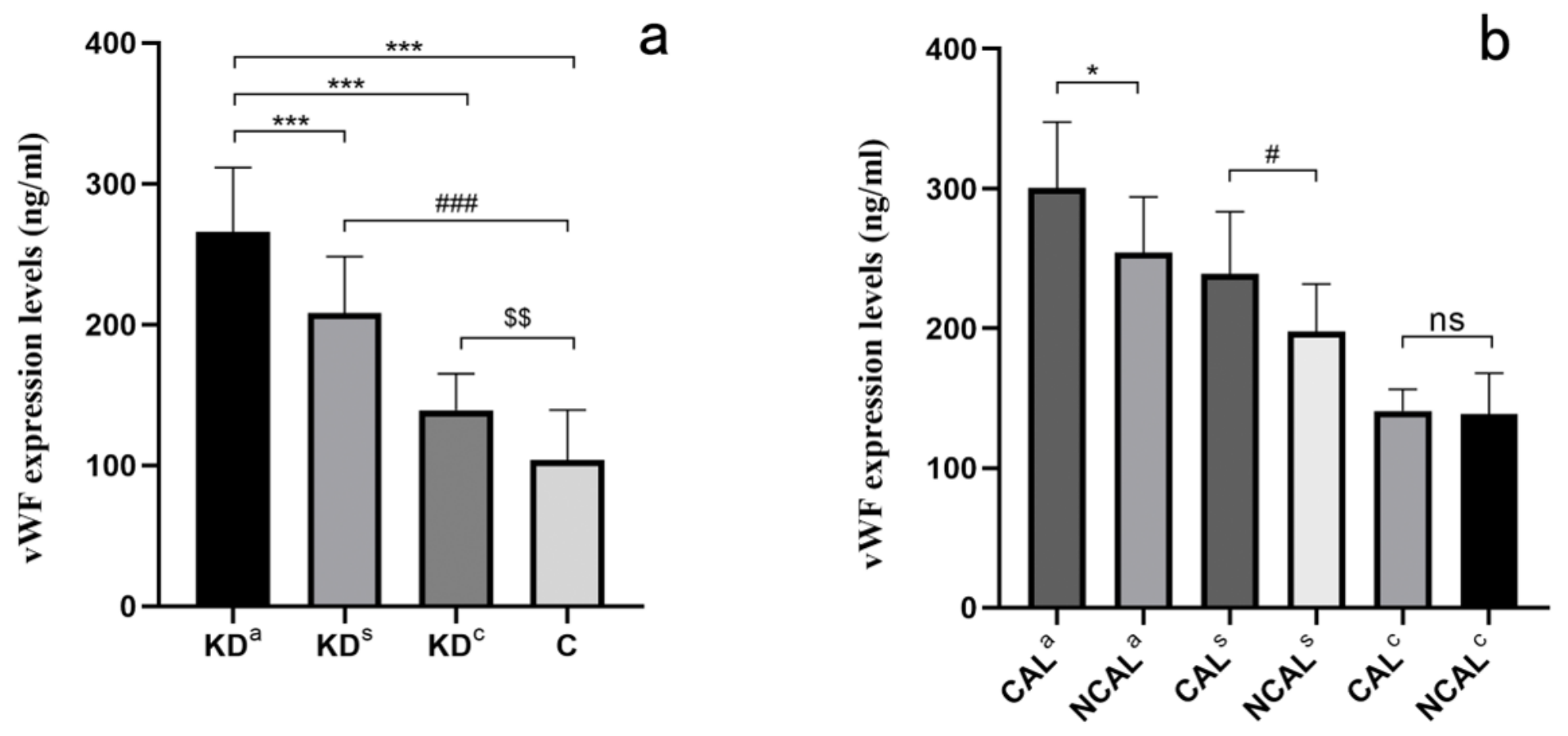

Figure 3

(a) Comparison of plasma vWF expression levels $(\mathrm{ng} / \mathrm{ml})$ between different groups. (b) Comparison of plasma vWF expression levels $(\mathrm{ng} / \mathrm{ml})$ between KD groups. */\#/\$P『0.05, **/\#\#/

$$
\mathrm{P}[0.01, * * * / \# \# \# /
$$

$\$ P \llbracket 0.001 ; n s, P \otimes 0.05$. 


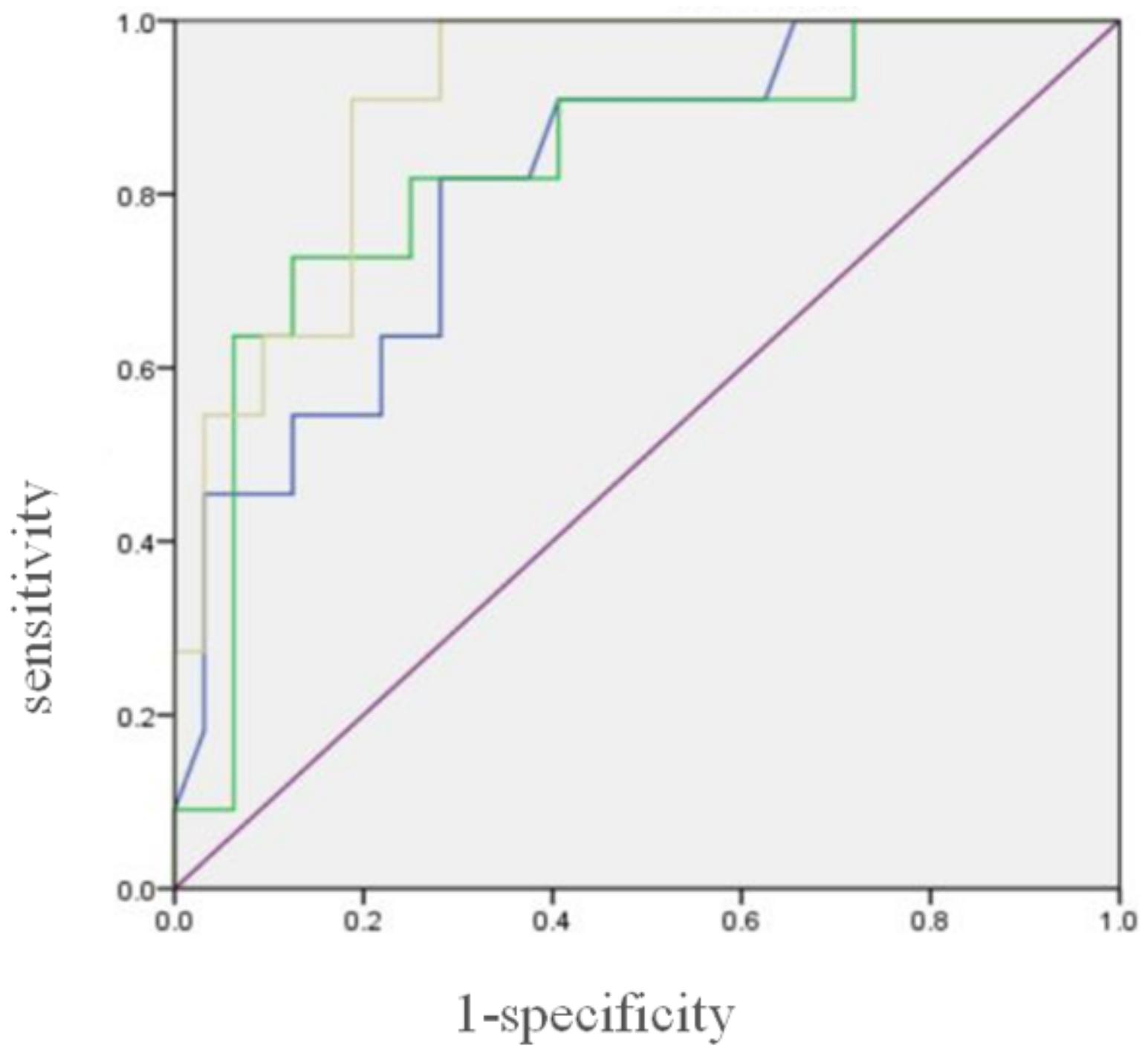

Figure 4

Individual factors and combined factors prediction of ROC curve of CALs. The blue line represents CD31+/CD42b-EMPs; The green line represents vWF; The yellow line represents CD31+/CD42bEMPs+vWF. 